\title{
INFLUÊNCIA DA INOCULAÇÃO DE CISTOS NA PRODUÇÃO DE BIOMASSADE "BRANCHONETA" Dendrocephalus brasiliensis PESTA, 1921 (CRUSTACEA: ANOSTRACA)
}

\author{
Influence of cysts inoculation in biomass production of Dendrocephalus \\ brasiliensis Pesta, 1921 (Crustacea: Anostraca)
}

\author{
José Patrocínio Lopes ${ }^{1}$, Hélio de Castro Bezerra Gurgel², Arrilton Araujo², Cibele Soares Pontes ${ }^{3}$
}

\begin{abstract}
RESUMO
Objetivou-se, neste trabalho desenvolver uma metodologia para produção de biomassa de "branchoneta" Dendrocephalus brasiliensis Pesta, 1921. Os experimentos foram realizados na Estação de Piscicultura da CHESF, Paulo Afonso-BA, com a finalidade de viabilizar a branchoneta como fonte alternativa de alimento na aqüicultura. A metodologia constou de dois tratamentos (com e sem inoculação de cistos de $D$. brasiliensis) realizada em duas épocas distintas (maio e outubro), com duas repetições. Foram utilizados quatro viveiros semi-escavados. Após a ANOVA $(\mathrm{P}<0,05)$ constatou-se que o tratamento com inoculação apresentou uma produção média de 18,63 $\pm 0,74 \mathrm{~kg}$ superior a $8,00 \pm 0,75 \mathrm{~kg}$ do outro tratamento, podendo-se assim produzir uma biomassa de $1.863 \mathrm{~kg} / \mathrm{ha} /$ ano. Os resultados obtidos recomendam produção de biomassa de $D$. brasiliensis utilizando a metodologia de inoculação de cistos.
\end{abstract}

Termos para indexação: Crustáceo, ovos de resistência, Thamnocephalidae.

\begin{abstract}
This work aimed to develop a methodology for the production of biomass of "branchoneta" Dendrocephalus brasiliensis Pesta, 1921. The experiments were accomplished at Paulo Afonso Fishculture Station of CHESF, BA, with the purpose of making possible the branchoneta as an alternative source of food in the aquaculture. The methodology consisted of two treatments (with and without inoculation of $D$. brasiliensis cysts) carried out at two different times (May and October), with two repetitions to each treatment. Four semi-excavated ponds, each with an area of $2000 \mathrm{~m}^{2}$, were used. After ANOVA $(\mathrm{P}<0,05)$ it was verified that treatment with inoculation showed the best results: $18,63 \pm 0,74 \mathrm{~kg}$ superior to $8,00 \pm 0,75 \mathrm{~kg}$ of the other treatment. This suggests an average biomass production of $1.863 \mathrm{~kg} / \mathrm{ha} /$ year. The results obtained recommend production of biomass of D. brasiliensis using the methodology of inoculation of cysts.
\end{abstract}

Index terms: Crustacea, resistance eggs, Thamnocephalidae.

(Recebido em 5 de dezembro de 2006 e aprovado em 2 de maio de 2007)

\section{INTRODUÇÃO}

$\mathrm{Na}$ aqüicultura praticada na atualidade, um dos maiores problemas na larvicultura de camarões e peixes é a demanda por um arraçoamento adequado (na forma de cistos, náuplios, larvas, alevinos ou juvenis de artêmia ou peixes) ou inerte (biomassa). A Artemia sp. é um alimento de sustentação na larvicultura de camarões e peixes, porém o alto custo para cistos GSL classe A, em torno de US\$ $100.00 / \mathrm{kg}$, aliado à escassez no mercado, restringe o crescimento do cultivo dessas espécies (CÂMARA, 2000).

A branchoneta, Dendrocephalus brasiliensis Pesta, 1921 (Crustacea, Anostraca) teve o ciclo reprodutivo completado na Estação de Piscicultura de Paulo Afonso (EPPA) de propriedade da Companhia Hidro Elétrica do
São Francisco (CHESF), e constitui uma importante fonte alternativa de alimento para larvas e alevinos das diversas espécies de peixes carnívoros (LOPES, 1998).

Lopes \& Tenório (2005) citam que, durante o acompanhamento da dieta alimentar de alevinos de Lophiosilurus alexandri Steindachner, 1876 em tanques com D. brasiliensis, mesmo mortos, já atraia todos os alevinos para o consumo imediato dos mesmos.

Lopes et al. (2006), comparando o crescimento (peso e comprimento) e sobrevivência durante 60 dias de cultivo, de um grupo de 90 alevinos da espécie Pterophyllum scalare Schultze, 1823, alimentados com D. brasiliensis e outro grupo de 90 alevinos da mesma espécie, alimentados com ração floculada para peixes ornamentais, encontraram

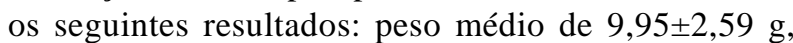

\footnotetext{
Doutor - Gerência Regional de Operação de Paulo Afonso/GRP - Companhia Hidro Elétrica do São Francisco/CHESF - Rua do Comando Usina II 48600-000 - Paulo Afonso, BA - jlopes@chesf.gov.br

${ }^{2}$ Doutores, Professores - Departamento de Fisiologia - Programa de Pós-Graduação em Psicobiologia - Universidade Federal do Rio Grande do Norte/ UFRN - Campus Universitário - Cx. P. 1511 - 59078-970 - Natal, RN - helio@cb.ufrn.br; arrilton@cb.ufrn.br

${ }^{3}$ Doutora - Departamento de Fisiologia - Universidade Federal Rural do Smi-Árido/UFERSA - KM 47, BR 110 - Presidente Costa e Silva - Cx. P. 137 59625-900 - Mossoró, RN - cibelepontes@yahoo.com.br
} 
comprimento médio de 94,64ะ13,02 mm e sobrevivência de $88 \%$ para os alevinos alimentados com D. brasiliensis e, peso médio de $3,30 \pm 0,77 \mathrm{~g}$, comprimento médio de $58,20 \pm 6,82 \mathrm{~mm}$ e sobrevivência de $53 \%$ para os alevinos alimentados com ração floculada.

O cultivo em massa de D. brasiliensis pode minimizar as dificuldades e aumentar a produtividade de alevinos, principalmente na larvicultura de peixes carnívoros (LOPES et al., 2007).

Objetivou-se, neste trabalho desenvolver uma metodologia de produção de biomassa do anostráceo $D$. brasiliensis em larga escala para utilização como alimento na aquiicultura.

\section{MATERIAL E MÉTODOS}

O experimento foi realizado na Estação de Piscicultura de Paulo Afonso (EPPA), (09²2'38" S e $\left.38^{\circ} 13^{\prime} 58^{\prime \prime} \mathrm{W}\right)$, localizada no município de Paulo Afonso, Bahia, pertencente à Companhia Hidro Elétrica do São Francisco (CHESF).

Foram selecionados quatro viveiros da EPPA, sendo escolhidos os viveiros de números 12, 13, 14 e 15. Os critérios adotados para seleção foram os seguintes: todos os quatro viveiros apresentavam em comum a forma retangular $\mathrm{e}$ medindo $40 \times 50 \mathrm{~m}$, profundidade média de $0,80 \mathrm{~m}$, localização em série e superfície inundada de $2.000 \mathrm{~m}^{2}$.

Os viveiros, após totalmente secos, foram limpos de vegetação aquática e efetuada a fertilização orgânica (esterco bovino), na proporção de $150 \mathrm{~g} / \mathrm{m}^{2}$

Para o preparo dos viveiros, eles foram expostos ao sol durante três dias, tiveram o solo revolvido para liberação de gases e secagem mais rápida.

Visando a produção de biomassa em grande escala, durante o enchimento inicial dos viveiros, os de no 12 e 13, foram inoculados com um grama de cistos de branchoneta para cada mil metros quadrados (LOPES et al., 2007). Os viveiros 14 e 15, não foram inoculados, esperou-se apenas a criação natural de branchonetas através dos cistos existentes no solo desses viveiros provenientes de cultivos anteriores. A partir desse ponto foi acompanhado todo seu desenvolvimento.

Como os viveiros apresentam a mesma área, porém profundidades variando entre 0,60 a 1,0 $\mathrm{m}$ com uma média de $0,80 \mathrm{~m}$ e, visando uniformizar as amostras de água, elas, foram coletadas em cinco pontos distintos: na caixa de coleta (próximo aos locais de abastecimento e de drenagem), nas profundidades de $0,50 \mathrm{~m}, 0,80 \mathrm{~m}$ e a $1,0 \mathrm{~m}$ e a cada dois dias, sendo posteriormente misturadas, homogeneizadas e acondicionadas em garrafas. Depois elas foram colocadas em freezer, para envio ao Laboratório de Limnologia da Universidade Federal Rural de Pernambuco (UFRPE), para análises físicas, químicas e biológicas, visando monitorar a qualidade da água, durante o período de cultivo, e conhecer as variáveis ideais para o desenvolvimento desse animal.

As amostras do material planctônico, depois de coletadas foram fixadas em formol a $4 \%$ e encaminhadas ao Laboratório de Plâncton do Departamento de Pesca da UFRPE para análise do fitoplâncton e zooplâncton. As amostras foram coletadas a partir de 60 litros de água filtrada, em rede cônico-cilíndrica de um metro de comprimento, com malha de $30 \mathrm{~mm}$ e copo próprio para acondicionamento do material concentrado, provido de uma torneira para facilitar a transferência do material para o pote das amostras. Estas foram fixadas em formol neutralizado e acondicionadas em potes de $250 \mathrm{~mL}$, de modo a obter-se uma concentração de $5 \%$, após o armazenamento.

Para monitoramento do oxigênio dissolvido, $\mathrm{pH}$ e temperatura da água foi utilizado um aparelho portátil, multiparâmetro de leitura direta da marca YSI 556 MPS.

Decorridos 15 dias do início do cultivo, quando todas branchonetas estavam adultas, e apresentavam os ovissacos repletos de cistos, foi efetuada a coleta desses animais, visando a produção de biomassa. Tendo em vista o comprimento total das branchonetas (entre 1,0 a 1,5 cm), na

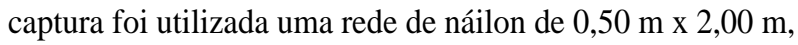
com malha de 1,0 mm. Não foi necessária a drenagem total da água dos viveiros, tendo em vista a facilidade de captura total dos animais que ficam sempre agrupados. Depois de coletados, foram transportados até o laboratório da EPPA e distribuídos em incubadoras de fibra de vidro com capacidade de 200 litros (Figura 1), com água corrente por 24 horas, para retirada de impurezas (lodo, algas filamentosas, folhas de macrófitas aquáticas etc.) provenientes dos viveiros.

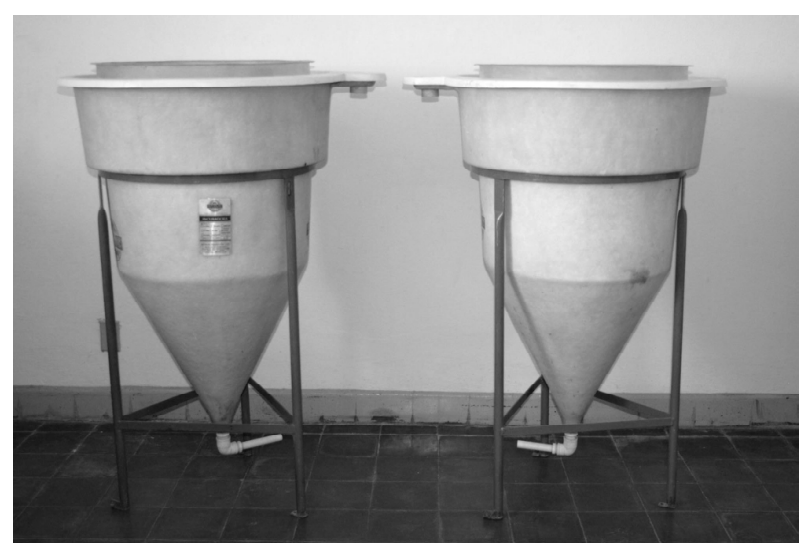

Figura 1 - Incubadoras utilizadas no trabalho. 
Cada incubadora recebeu em torno de cinco quilos de branchoneta. Quando não mais se observavam impurezas na água, com uso de uma mangueira de $3 / 4$ foi realizada a transferência das branchonetas para baldes vazios, acoplados com uma tela, para evitar a fuga desses crustáceos durante a drenagem da água. A seguir, a biomassa foi pesada em balança mecânica com capacidade para $15 \mathrm{~kg}$ e efetuada a distribuição em sacolas de plástico, formando tabletes de um quilo e colocadas em freezer para posterior alimentação das espécies de peixes carnívoros, na fase de alevinos cultivadas na EPPA.

Para análise estatística, considerou-se o desenho experimental $2 \times 2 \times 2$, dois níveis de inoculação (tratamentos), duas épocas do ano (inverno e verão) para verificar influência desses fatores climáticos e duas repetições, perfazendo um total de oito unidades experimentais. O modelo matemático (GOMES, 1987) é representado por:

$$
\begin{aligned}
& \frac{\bar{X}}{X}=\mathrm{m}+\mathrm{I}+\mathrm{E}+\mathrm{I} . \mathrm{E}+\mathrm{e}, \text { onde: } \\
& \mathrm{m}=\text { Mariável resposta }(\mathrm{kg} \text { de biomassa/ha/ano }) \\
& \mathrm{I}=\text { Efeito dos modelo matemático }
\end{aligned}
$$
cistos)

$\mathrm{E}=$ Efeito das épocas do ano (maio e outubro)

I . E = Efeito da interação nível de inoculação : época do ano

$$
\mathrm{e}=\text { Erro experimental } \text {. }
$$

Após os experimentos aplicou-se a Análise de Variância (ANOVA), com $\mathrm{P}<0,05$ onde os resultados calculados se confrontaram com os resultados tabelados (Tabela de Fisher).

\section{RESULTADOS E DISCUSSÃO}

Os resultados das análises químicas (ortofosfato, amônia, nitrito, nitrato, alcalinidade e dureza total), apresentam-se na tabela 1 .

As concentrações de oxigênio dissolvido na água dos viveiros, ao longo do período de cultivo, apresentaram os seguintes valores médios: 7,98 $\pm 0,50 ; 8,94 \pm 0,54 ; 8,52 \pm 0,64$ e $8,88 \pm 0,53 \mathrm{mg} / \mathrm{L}$ para os viveiros $12,13,14$ e 15 , respectivamente.

Os valores de $\mathrm{pH}$ da água foram alcalinos em todos os viveiros, apresentando valores compreendidos entre 7,85 e 9,25 para o viveiro $12 ; 7,85$ e 9,30 para o viveiro 13 ; 8,50 e 8,80 para o viveiro 14 e 8,25 e 8,95 para o viveiro 15 , com médias de $8,42,8,42,8,57$ e 8,35 para os viveiros 12,13 , 14 e 15 respectivamente.

Com referência às temperaturas máximas diárias das águas dos viveiros no $12,13,14$ e 15, observou-se uma temperatura média de $31^{\circ} \mathrm{C}$ logo no início do cultivo, caindo gradativamente e estabelecendo-se em torno de $28{ }^{\circ} \mathrm{C}$ até

\begin{tabular}{|c|c|c|c|c|c|c|c|c|c|c|c|c|}
\hline \multirow[t]{2}{*}{$\begin{array}{l}\text { Variável/ } \\
\text { Viveiro }\end{array}$} & \multicolumn{2}{|c|}{$\begin{array}{c}\text { Ortofosfato } \\
\mu \mathrm{g} / \mathrm{L}\end{array}$} & \multicolumn{2}{|c|}{$\begin{array}{l}\text { Amônia } \\
\mu \mathrm{g} / \mathrm{L}\end{array}$} & \multicolumn{2}{|c|}{$\begin{array}{l}\text { Nitrito } \\
\mathrm{mg} / \mathrm{L}\end{array}$} & \multicolumn{2}{|c|}{$\begin{array}{l}\text { Nitrato } \\
\mu \mathrm{g} / \mathrm{L}\end{array}$} & \multicolumn{2}{|c|}{$\begin{array}{l}\text { Alcalinidade } \\
\mathrm{mg} / \mathrm{L} \mathrm{CaCO}_{3}\end{array}$} & \multicolumn{2}{|c|}{$\begin{array}{c}\text { Dureza } \\
\mathrm{mg} / \mathrm{L} \mathrm{Ca}\end{array}$} \\
\hline & Maio & Out. & Maio & Out. & Maio & Out. & Maio & Out. & Maio & Out. & Maio & Out. \\
\hline 12 & $\begin{array}{c}215,28 \\
\pm\end{array}$ & $\begin{array}{c}13,62 \\
\pm\end{array}$ & $\begin{array}{c}25,53 \\
\pm\end{array}$ & $\begin{array}{c}4,20 \\
\pm\end{array}$ & $\begin{array}{c}0,10 \\
\pm\end{array}$ & $\begin{array}{c}0,10 \\
\pm\end{array}$ & $\begin{array}{c}0,21 \\
\pm\end{array}$ & $\begin{array}{r}0,45 \\
\pm\end{array}$ & $\begin{array}{c}50,88 \\
\pm\end{array}$ & $\begin{array}{c}38,00 \\
\pm\end{array}$ & $\begin{array}{c}10,19 \\
\pm\end{array}$ & $\begin{array}{c}10,19 \\
\pm\end{array}$ \\
\hline & 10,15 & 02,86 & 07,07 & 0,20 & 0,15 & 0,12 & 0,10 & 0,10 & 02,55 & 04,21 & 01,15 & 01,12 \\
\hline 13 & $\begin{array}{c}252,20 \\
\pm\end{array}$ & $\begin{array}{c}25,00 \\
\pm\end{array}$ & $\begin{array}{l}94,30 \\
\pm\end{array}$ & $\begin{array}{c}1,78 \\
\pm\end{array}$ & $\begin{array}{c}0,62 \\
\pm\end{array}$ & $\begin{array}{c}0,15 \\
\pm\end{array}$ & $\begin{array}{c}0,30 \\
\pm\end{array}$ & $\begin{array}{c}0,26 \\
\pm\end{array}$ & $\begin{array}{c}52,08 \\
\pm\end{array}$ & $\begin{array}{c}25,00 \\
\pm\end{array}$ & $\begin{array}{c}10,79 \\
\pm\end{array}$ & $\begin{array}{c}06,70 \\
\pm\end{array}$ \\
\hline & 31,02 & 11,17 & 10,22 & 0,11 & 0,10 & 0,10 & 0,12 & 0,09 & 02,10 & 01,54 & 0,12 & 01,13 \\
\hline 14 & $\begin{array}{c}136,19 \\
\pm\end{array}$ & $\begin{array}{c}140,00 \\
\pm\end{array}$ & $\begin{array}{c}70,86 \\
\pm\end{array}$ & $\begin{array}{c}3,50 \\
\pm\end{array}$ & $\begin{array}{c}0,20 \\
\pm\end{array}$ & $\begin{array}{r}0,21 \\
\pm\end{array}$ & $\begin{array}{r}0,39 \\
\pm\end{array}$ & $\begin{array}{r}0,43 \\
\pm\end{array}$ & $\begin{array}{c}45,10 \\
\pm\end{array}$ & $\begin{array}{c}48,08 \\
\pm\end{array}$ & $\begin{array}{c}9,49 \\
\pm\end{array}$ & $\begin{array}{c}12,20 \\
\pm\end{array}$ \\
\hline & 28,82 & 10,60 & 10,43 & 0,12 & 0,05 & 0,05 & 0,02 & 0,02 & 01,20 & 02,21 & 0,10 & 0,10 \\
\hline 15 & $\begin{array}{c}77,17 \\
\pm\end{array}$ & $\begin{array}{l}\mid 35,00 \\
\quad \pm\end{array}$ & $\begin{array}{c}25,54 \\
\pm\end{array}$ & $\begin{array}{c}4,50 \\
\pm\end{array}$ & $\begin{array}{c}0,23 \\
\pm\end{array}$ & $\begin{array}{r}0,05 \\
\pm\end{array}$ & $\begin{array}{r}0,01 \\
\pm\end{array}$ & $\begin{array}{c}0,49 \\
\pm\end{array}$ & $\begin{array}{c}0,62 \\
\pm\end{array}$ & $\begin{array}{c}49,50 \\
\pm\end{array}$ & $\begin{array}{c}11,27 \\
\pm\end{array}$ & $\begin{array}{c}12,20 \\
\pm\end{array}$ \\
\hline & 03,42 & 01,24 & 01,22 & 0,05 & 0,04 & 0,01 & 0,008 & 0,02 & 0,01 & 07,43 & 0,10 & 0,10 \\
\hline
\end{tabular}
o final do experimento.

A análise do plâncton para os quatro viveiros visando-se identificar os prováveis tipos de alimentos a

Tabela 1 - Resultados das variáveis químicas da água dos viveiros de cultivo por período estudado. 
serem utilizados por D. brasiliensis, nessa primeira etapa (maio), para os quatro viveiros, apresentaram os seguintes resultados: com relação ao fitoplâncton, demonstraram que existe uma diferença entre a flora algológica dos viveiros 12 e 13, do ponto de vista quantitativo. O viveiro 12, nesse período, esteve representado pelas seguintes Divisões: Chlorophyta com quatro espécies; Cyanophyta com a espécie Oscillatoria communis e Bacyllariophyta com as espécies Fragillaria crotonensis, Melosira sulcata, Pinularia sp. e Surirela sp. O viveiro 13 apresentou apenas a Divisão Chlorophyta com as espécies Arthosdesmus identatus, Cosmarium humile, Pediastrum simplex e Planktosphoria gelatinosa. Com relação aos viveiros 14 e 15 apresentaram-se, nesse período, quantitativamente inferiores aos viveiros 12 e 13 . O viveiro 13 apresentou apenas três espécies de Chlorophyta e uma de Bacyllariophyta. O viveiro 14 apresentou três espécies de Chlorophyta somente no final do cultivo. A Cyanophyta Oscillatoria e as Bacillariophytas Fragillaria crotonensis, Melosira sulcata e Surirela sp. também estiveram presentes somente no final do cultivo.

No mês de outubro, os viveiros apresentaram uma baixa diversidade de espécies fitoplanctônicas havendo flutuações de uma maneira geral, estando representados pelas Divisões Chlorophyta Cyanophyta e Bacyllariophyta. Com relação ao zooplâncton - Os grupos da comunidade zooplanctônica, identificados nos viveiros, para os dois períodos, estiveram representados por Copepoda, Cladocera, Ostracoda e Rotifera. Os copépodos foram mais representativos com um número de 60 a 260 org./L, sendo seguido pelos cladóceros, com um número de 15 a 98 org./ L. Os rotiferos e ostracodas foram menos representativos variando de 2 a 5 org./L e 2,9 a 11,6 org/L, respectivamente.

O total de biomassa obtido foi de $65 \mathrm{~kg}$ durante o ciclo de 15 dias de cultivo do mês de maio e de $41,5 \mathrm{~kg}$, durante os 15 dias de cultivo do mês de outubro. Pelos resultados obtidos verificou-se que é possível produzir em torno de $2.000 \mathrm{~kg}$ de biomassa de branchoneta por hectare/ano.

A produção média de biomassa em viveiros inoculados foi de $18,63 \pm 0,75 \mathrm{~kg} /$ viveiro/ciclo, superior aos produzidos nos viveiros não inoculados que foi de $8,0 \pm 0,75$ $\mathrm{kg} /$ viveiro/ciclo. Os resultados para o tratamento com inoculação diferiram entre épocas, observando-se uma melhor produção no mês de maio, com média de 16,25 $\pm 0,75$ $\mathrm{kg}$ /viveiro/ciclo superior ao mês de outubro que foi de $10,38 \pm 0,75 \mathrm{~kg} /$ viveiro/ciclo. Tendo em vista a interação Inoculação:Época, para produção de biomassa, ressaltase que a Inoculação/Maio, apresentou melhor resultado com 22,25 $\pm 0,96 \mathrm{~kg} /$ viveiro/ciclo, que foi superior às outras

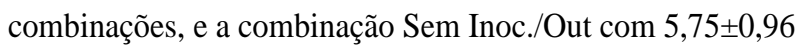
$\mathrm{kg}$ foi a que apresentou menor representatividade.

Aplicando a Análise de Variância (ANOVA), com $\mathrm{P}<0,05$ do modelo fatorial para a produção de biomassa (kg/viveiro/ciclo), verificou-se que houve diferença significativa para o fator de variação Inoculação $(\mathrm{P}<0,0002)$, existindo também diferença significativa para o fator Época ( $\mathrm{P}<0,0026)$.

A ANOVA $(\mathrm{P}<0,05)$ indicou que houve diferença significativa $(\mathrm{P}<0,001)$ para a interação Inoculação: Época, o que quer dizer que existiu efeito para combinação Inoculação:Época para produção de biomassa.

$\mathrm{O}$ ortofosfato $\left(\mathrm{PO}_{4}\right)$, nos viveiros 12 e 13 em estudo apresentou concentrações altas, com valores compreendidos entre 215,28 e $252,60 \mu \mathrm{g} / \mathrm{L}$ no início do cultivo e com valores semelhantes ao final. Os viveiros 14 e 15 apresentaram no início do cultivo valores de 136,19 e $77,17 \mu \mathrm{g} / \mathrm{L}$ e 95,42 e 33,30 $\mu \mathrm{g} / \mathrm{L}$ no final, respectivamente. Os maiores valores desse fósforo, nos viveiros 12 e 13 deveram-se provavelmente aos solos desses viveiros serem mais argilosos, influenciando na maior concentração de matéria orgânica.

Segundo Chen \& Chin (1988), citados por Vinatea (1997), a concentração de amônia aceitável para pós-larvas de camarão é de $480 \mu \mathrm{g} / \mathrm{L}$ de amônia total. A concentração de amônia da água dos viveiros em estudo apresentou valores inferiores com uma média de $58,30 \mu \mathrm{g} / \mathrm{L}$, portanto sem riscos de causar prejuízos aos animais em estudo. Ainda segundo Chen \& Chin (1988), citados por Vinatea (1997), o valor aceitável de nitrito para pós-larvas de camarão é de $0,57 \mathrm{mg} / \mathrm{L}$. A concentração de nitrito, em todos os viveiros, no período de cultivo foi de 0,10 a $0,62 \mathrm{mg} / \mathrm{L}$, com uma média de $0,22 \mathrm{mg} / \mathrm{L}$, dentro de faixas aceitáveis para aqüicultura.

Quanto ao nitrato $\left(\mathrm{NO}_{3}\right)$, as concentrações foram baixas em todos os viveiros. A alcalinidade e a dureza total da água dos viveiros estudados, durante o período de cultivo, mantiveram-se entre 41 a $63 \mathrm{mg} / \mathrm{L}$ de $\mathrm{CaCO}_{3}$ Essa estabilidade foi observada em todos os viveiros envolvidos durante o experimento.

Os valores de oxigênio dissolvido corresponderam a uma condição compatível com as necessidades metabólicas dos organismos aquáticos, pois segundo Sipaúba-Tavares (1995), valores acima de $4 \mathrm{mg} / \mathrm{L}$ apresentam boas condições para criação de organismos aquáticos.

$\mathrm{O}$ pH no período do cultivo permaneceu na faixa compreendida entre 8 a 8,5, não sendo ideal para Rotifera, Copepoda e Cladocera, segundo Sipaúba-Tavares \& Rocha (2001). No entanto nessa faixa de pH, teve-se um bom desenvolvimento de $D$. brasiliensis. 
Quanto à temperatura da água, observou-se que ela permaneceu entre 26 a $31^{\circ} \mathrm{C}$, havendo um aumento no número de Copepoda, Cladocera e Rotifera, estando os resultados de acordo com as faixas ideais de temperatura para esses animais, de acordo com as descritas por SipaúbaTavares \& Rocha (2001). Tais temperaturas coincidem com as identificadas por Walsche et al. (1991) como as melhores para a espécie Streptocephalus proboscideus Frauenfeld, 1873 da mesma família de D. brasiliensis, segundo Gonçalves (2001).

Aos 13 dias de cultivo observou-se redução no número de organismos do plâncton. Essa redução pode estar associada ao ciclo de vida curto de muitos dos organismos ou também com a diminuição do alimento pela ação filtradora da branchoneta (LOPES, 1998).

Os resultados na produção de biomassa poderiam ter sido mais promissores, contudo os viveiros 14 e 15 (sem inoculação) produziram uma boa quantidade de biomassa, 11 e 9,5 kg no mês de maio e 6,5 e 5,0 kg no mês de outubro respectivamente. Porém, nessa biomassa, os animais apresentavam comprimentos médios inferiores a $1,0 \mathrm{~cm}$, isso em razão da grande quantidade de branchonetas nascidas naturalmente nesses viveiros $-\mathrm{em}$ torno de 1.000 .000 de animais $\left(>500 / \mathrm{m}^{2}\right)$ - prejudicando a produção final de biomassa. Nos viveiros 12 e 13 (com inoculação) em vista da quantidade de animais produzidos $\left(<500 / \mathrm{m}^{2}\right)$ através do controle de inoculação de cistos, gerou-se uma população de animais bem desenvolvidos, cujos comprimentos médios foram superiores a $1,5 \mathrm{~cm}, \mathrm{o}$ que influenciou na maior produção de biomassa, em relação aos viveiros não inoculados.

O melhor resultado de biomassa obtido foi com inoculação, no mês de maio, com 65 quilos superando a expectativa que era de $60 \mathrm{~kg}$ para os quatros viveiros com $15 \mathrm{~kg} /$ viveiro/15 dias de cultivo. Quando da combinação Inoculação:Época a que apresentou também melhor resultado foi Inoculação/Maio com 22,25 $\pm 0,96 \mathrm{~kg} /$ viveiro/ ciclo, superior as demais combinações. Tal resultado pode estar associado ao fator inverno ocorrido nesse período, influenciando na riqueza das águas através do aporte de nutrientes e contribuindo com a maior diversidade de organismos do plâncton, conforme resultados das análises. Como houve diferença significativa entre as duas melhores médias das combinações, ao nível de $\mathrm{P}<0,05$ e ambas com inoculação, para uma melhor representatividade média da produção por ciclo ao longo do ano, utilizou-se para efeito de cálculo da produção por hectare/ano, a média do tratamento com inoculação, independentemente da época, $(16,25 \pm 0,75)$.
Esses resultados são ínfimos quando se contrasta com os obtidos por Vinatea (1997), citado por Vinatea (1999) com Artemia franciscana (cepa de Macau), em cultivos realizados no município de Acaraú (CE, Brasil). Considerando que esses foram os primeiros resultados alcançados nesse sistema semi-intensivo, onde não foram utilizadas rações ou suplementos alimentares na alimentação desses anostráceos mas somente alimento natural, vislumbra-se nesse microcrustáceo uma excelente fonte alternativa de alimento, para utilização na aqüicultura.

Esse trabalho é de suma importância para a região semi-árida do Nordeste Brasileiro, onde essa produção é obtida através de uma tecnologia simples, tornando o sistema de cultivo bastante atrativo para a larvicultura de peixes, camarões e de outros animais aquáticos cultiváveis. Quanto à biomassa de branchonetas, em virtude de sua alta qualidade nutricional, com níveis de proteína em torno de 67\% (LOPES, 1998), quando limpa de impurezas e congelada pode ser comercializada a US $\$ 3.00 / \mathrm{kg}$, tendo como principais clientes laboratórios de produção de larvas de camarões, aquariofilistas, ranicultores e criadores de peixes carnívoros.

Dessa forma, nas condições testadas (com inoculação e sem inoculação) e respaldadas nas análises estatísticas (ANOVA), é possível uma produção aproximada de $2.000 \mathrm{~kg}$ de biomassa/ha/ano, considerando vinte ciclos anuais e 66 dias para a preparação dos viveiros. A produção $5.764 \mathrm{~kg} / \mathrm{ha} / \mathrm{ano}$ de biomassa de Artemia sp. cultivada em tanques de 0.17 ha, obtida por Castro (1993), citado por Vinatea (1999) no México, mostra-se significativamente superior. No entanto, classifica-se esse resultado como "promissor" ao se registrar a produção de $1.863 \mathrm{~kg} / \mathrm{ha} / \mathrm{ano}$ de biomassa (peso úmido).

\section{CONCLUSÃO}

Concluiu-se que é possível, ao se inocular cada viveiro de $2.000 \mathrm{~m}^{2}$ com $2 \mathrm{~g}$ de cistos de D. brasiliensis, obter $1.863 \mathrm{~kg}$ de biomassa por hectare/ano, para utilização na aqüicultura, em especial na piscicultura e carcinicultura, através de uma tecnologia de fácil transferência e de baixo custo para os produtores.

\section{REFERÊNCIAS BIBLIOGRÁFICAS}

CÂMARA, M. R. Artemia no Brasil: do extrativismo ao cultivo. Revista Panorama da Aqüicultura, [S.1.], v. 10, n. 62, p. 15-19, 2000.

GOMES, F. P. Curso de estatística experimental. 12. ed. São Paulo: Nobel, 1987. 467 p. 
GONÇALVES, J. L. Remoção de algas via alimentação pelo microcrustáceo Dendrocephalus brasiliensis (Crustacea: Anostraca). 2001. Dissertação (Mestrado em Tecnologias Ambientais) - Universidade Federal de Mato Grosso do Sul, Campo Grande, 2001.

LOPES, J. P. Considerações sobre a branchoneta, Dendrocephalus brasiliensis, (Crustacea, Anostraca, Thamnocephalidae) como fonte alternativa na alimentação de alevinos Espécies Carnívoras. 1998. Monografia (Especialização em Aqüicultura) - Universidade Federal Rural de Pernambuco, Recife, 1998.

LOPES, J. P.; GURGEL, H. C. B.; GÁLVEZ, A. O.; PONTES, C. S. Produção de cistos de "branchoneta" Dendrocephalus brasiliensis (Crustacea: Anostraca). Revista Biotemas, [S.1.], v. 20, n. 2, p. 33-39, 2007.

LOPES, J. P.; PONTES, C. S.; ARAÚJO, A. A branchoneta na piscicultura ornamental. Revista Panorama da aquiicultura, Rio de Janeiro, v. 16, n. 94, p. 33-37, mar./ abr. 2006.

LOPES, J. P.; TENÓRIO, R. A. A branchoneta (Dendrocephalus brasiliensis, Pesta 1921) como fonte de alimento para alevinos de niquim (Lophiosilurus alexandri
Steindachner, 1876). Revista Nordestina de Zoologia, Maceió, v. 2, n. 1, p. 34-46, set. 2005.

SIPAÚBA-TAVARES, L. H. Limnologia aplicada à aqüicultura. Jaboticabal: Unesp, 1995. 71 p.

SiPAÚBA-TAVARES, L. H.; ROCHA, O. Produção de plâncton (Fitoplâncton e Zooplâncton) para alimentação de organismos aquáticos. São Carlos: RiMa, 2001. 106 p.

VINATEA, L. A. Princípios químicos da qualidade da água em aqüicultura. Florianópolis: UFSC, 1997. 231 p.

VINATEA, L. A. Manual de producción de artemia (quistes y biomassa) en módulos de cultivo: proyeto II-a/2 "localization, caracterización y evaluación del potencial extractivo de Artemia en Ibero - America con Destino a la Acuicultura". México: Universidad Autónoma Metropolitana/Unidad Xochimilco división de Ciencias Biológicas y de la Salud, 1999. $66 \mathrm{p}$.

WALSCHE, D. C.; MERTENS, J.; DUMONT, J. H. Observations on temperature optimum, cyst prodution and survival of Streptocephalus proboscideus (Frauenfeld 1873) (Crustracea: Anostraca), fed different diets. Hydrobiologia, [S.1.], v. 212, p. 31-26, 1991. 